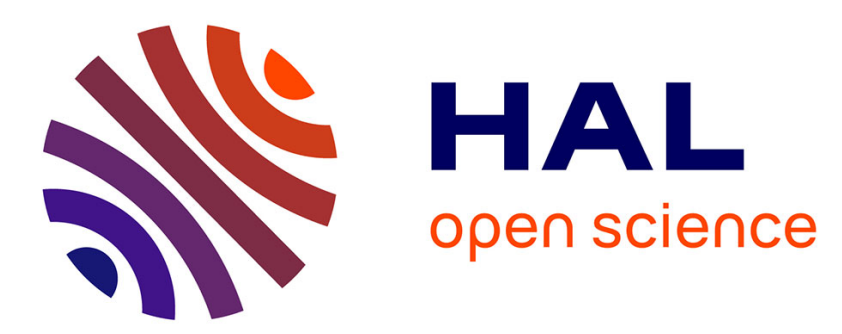

\title{
Influence of statistician involvement on reporting of randomized clinical trials in medical oncology
}

Julien Péron, Benoit You, Hui K Gan, Denis Maillet, Eric X Chen, Gregory R Pond

\section{- To cite this version:}

Julien Péron, Benoit You, Hui K Gan, Denis Maillet, Eric X Chen, et al.. Influence of statistician involvement on reporting of randomized clinical trials in medical oncology. Anticancer Drugs, 2013, 24, pp.306-309. 10.1097/CAD.0b013e32835c3561 . hal-02282697

\section{HAL Id: hal-02282697 https://hal.science/hal-02282697}

Submitted on 31 Aug 2020

HAL is a multi-disciplinary open access archive for the deposit and dissemination of scientific research documents, whether they are published or not. The documents may come from teaching and research institutions in France or abroad, or from public or private research centers.
L'archive ouverte pluridisciplinaire $\mathbf{H A L}$, est destinée au dépôt et à la diffusion de documents scientifiques de niveau recherche, publiés ou non, émanant des établissements d'enseignement et de recherche français ou étrangers, des laboratoires publics ou privés. 


\section{Influence of statistician involvement on reporting of randomized clinical trials in medical oncology Julien Péron ${ }^{\mathrm{a}, \mathrm{b}}$, Benoit You ${ }^{\mathrm{a}}$, Hui K. Gan ${ }^{\mathrm{c}, \mathrm{d}}$, Denis Maillet ${ }^{\mathrm{a}}$, Eric X. Chen ${ }^{\mathrm{e}}$ and Gregory R. Pond ${ }^{f}$}

Ideally, statisticians should be involved in the design, analysis, and reporting of randomized clinical trials (RCTs). This study assessed the impact of a statistician involvement in published medical oncology RCTs between 2005 and 2009. The reporting quality of each publication was rated using the Overall Reporting Quality Score on the basis of either 2001 or 2010 Consolidated Standards of Reporting Trials criteria. A four-question email survey on the statistical design and analysis was sent to the corresponding authors of each trial. Nonresponders were approached a maximum of three times. Overall, 107 responses were received from 357 solicited authors (30\%). Corresponding authors from industry-funded RCTs were less likely to respond ( 51 vs. $65 \%, P=0.013$ ). The same person was responsible for statistical design and analyses in $47 \%$ of cases. Overall, the statistician involved held a PhD (or equivalent) in statistics in most cases.

The statisticians responsible for the statistical design and analysis were listed as coauthors in 68 and $81 \%$ of RCT manuscripts. There was no statistically significant impact on manuscript reporting quality of the degree of statistician involvement in manuscript preparation. Fewer trials were reported as positive when the responsible statistician was listed as a coauthor. It is possible that RCTs included in this review are in general of higher quality or were more likely to have a greater level of statistician involvement than smaller, single-arm, or unpublished studies. This imbalance could explain the lack of significant difference observed in the Overall Reporting Quality Score between trials where statisticians were listed as coauthors or not.

Anti-Cancer Drugs 2013, 24:306-309

Keywords: methods, neoplasms, randomized-controlled trials as topic, research designs, research standards, statistics as topic

aDepartment of Medical Oncology, Centre Hospitalier Lyon-Sud, Hospices Civils de Lyon, Pierre-Bénite, ${ }^{b} \mathrm{CNRS}$, UMR 5558, Biometry and Evolutionary Biology Laboratory, Biostatistics Team, Villeurbanne, France, ' $J o i n t$ Austin-Ludwig Oncology Unit, Austin Hospital, ${ }^{\mathrm{d}}$ Cancer Trials Australia, Melbourne, Victoria, Australia, ${ }^{\mathrm{e}}$ Department of Medical Oncology and Hematology, Princess Margaret Hospital, University Health Network, Toronto and ${ }^{f}$ Ontario Clinical Oncology Group, Department of Oncology, McMaster University, Hamilton, Ontario, Canada

Correspondence to Julien Péron, Department of Biostatistics, Centre Hospitalier Lyon-Sud, Hospices Civils de Lyon, 165 chemin du grand revoyet, 69495 Pierre-Bénite, France

Tel: +33 61625 8991; fax: + 3347886 5774; e-mail: julien.peron@ymail.com

\section{Introduction}

Positive results from randomized-controlled trials (RCTs) often establish new standards of care and form the basis of regulatory approval of new drugs. It is therefore important that RCTs are well designed and conducted, but it is equally important that they are well reported. We and others have identified deficiencies in the reporting of RCT results. Among them, one of the most common deficiencies is the lack of reporting of key statistical parameters, such as the sample size calculation [1-5], the method in determining random allocation, the use of blinding, and the method of allocation concealment [5].

The involvement of a qualified statistician in the design, analysis, and reporting of RCTs can potentially address these deficiencies [6-8]. However, the degree of the statistician's involvement is difficult to ascertain in many RCT reports. As a potential, unvalidated, surrogate for statistician involvement, one could use the level of contribution to the final publication, as indicated by coauthorship. In general, journals require, as a policy, that all coauthors have substantially contributed to the performance of the study or analysis, the writing of the manuscript, and final approval of the manuscript [9]. It may be hypothesized that manuscripts that include as a coauthor the qualified statistician responsible for the design and analysis of the trial may have improved reporting of the clinical trial, particularly in the reporting of the key statistical parameters.

We conducted a survey of corresponding authors of RCTs publications to determine the extent of the involvement of a statistician and associate this with the quality of each publication. The primary outcome selected was the Overall Reporting Quality Score (OQS) [2,4,5] on the basis of either 2001 or 2010 Consolidated Standards of Reporting Trials (CONSORT) criteria $[10,11]$.

\section{Materials and methods}

The database was originally constructed to examine the adherence of recent publications of oncology RCTs to the CONSORT guidelines [5]. Eligible publications were 
phase III RCTs with $100+$ patients per arm. RCTs assessing systemic anticancer therapies published between January 2005 and December 2009 in 10 journals that were believed to publish the majority of oncology RCTs: Annals of Oncology; British Journal of Cancer; Breast Cancer Research and Treatment; Cancer; European Journal of Cancer; Journal of Clinical Oncology; Journal of the National Cancer Institute; Lancet; Lancet Oncology; and New England Journal of Medicine [5]. Studies were excluded from these reviews if they were pediatric studies ( $<18$ years of age); involved treatment solely with radiotherapy or surgery; phase I, II, or phase IV trials; supportive care, palliative care, hematological malignancy or prevention trials; metaanalyses, overviews, or publications using pooled data from two or more trials; and secondary reports of previously published trials.

For this study, an e-mail questionnaire was sent to the corresponding author of each publication. The questionnaire included a short message describing our objectives and previous findings (see Appendix) and four questions about the person in charge of the statistical analyses and design and their highest qualification in statistics. The questionnaire was deliberately brief in an attempt to maximize the number of respondents. If the corresponding author's email address was no longer current, a subsequent internet search of alternate contact details was carried out. If no valid address could be found, that author was considered noncontactable. If a response was not received within 30 days of the first email, two subsequent emails were sent at monthly intervals before that author was deemed a nonrespondent.

The reporting quality of each publication was rated using the 2001 and 2010 OQS, as described previously [5]. In brief, each item that was adequately reported was assigned one point. The overall OQS score was the sum of the points obtained, with maximum scores of 18 and 27 for CONSORT 2001 and 2010, respectively.

Descriptive statistics were calculated. Proportions were compared using $\chi^{2}$-tests. Continuous variables were compared using the Mann-Whitney $U$-test. Dichotomization of outcomes was carried out for increased statistical power. All tests were two sided, statistical significance was defined at the $\alpha$ level equal to 0.05 , and analyses were carried out using SAS version 9.1 (SAS Institute Inc., Cary, North Carolina, USA).

All questionnaires were sent out between 1 August and 31 October 2011 to authors of RCTs identified in previous reviews [5,12]. The characteristics of the trials studied have been described previously [5].

\section{Results}

Of 357 corresponding authors, $37(10 \%)$ had invalid contact details, with an internet search of updated contact details leading to updated contact information for 16 authors, whereas 21 remained noncontactable. Two of the 16 authors with updated contact information provided a response to the questionnaire, whereas the remaining 14 were nonrespondents. In total, 107 authors responded to the survey. Trial characteristics were statistically similar between those who responded and those who did not, except responders who were less likely to be industry funded (51 vs. $65 \%, P=0.013$ ) (Table 1 ).

All 107 respondents identified an investigator primarily responsible for the statistical design and analysis of the trial. The same investigator was responsible for both tasks in $50(47 \%)$ RCTs. In most cases, the responsible statistical investigator had a $\mathrm{PhD}$ (or equivalent) degree in statistics $(n=78,73 \%$ for study design; and $n=72$, $67 \%$ for statistical analysis).

The statistician responsible for the statistical analysis was included as a coauthor in 87 (81\%) manuscripts. However, statisticians responsible for the trial design were included as coauthors in only $73(68 \%)$ of manuscripts. The median OQS score did not differ significantly between those studies where the statistician (responsible for either design or analysis) was a coauthor or by the frequency of a $\mathrm{PhD}$-equivalent trained statistician (Table 2). Similar results were observed (data not shown) when only those CONSORT items deemed directly related to the statistical methods were included, such as sample size calculation, method in determining random allocation, the use of blinding, and the method of allocation concealment. However, a trend toward fewer positive trials (according to the primary endpoint) was observed when the statisticians primarily responsible in the design ( 47 vs. $65 \%, P=0.066)$ or analysis ( 47 vs. $70 \%$, $P=0.084)$ were included as coauthors.

\section{Discussion}

This study is the first to quantify the frequency and impact of statistical input into the reporting quality of RCTs. Reassuringly, all trials could identify an investigator who was responsible for the statistical design and analysis. Attempts to quantify the degree of their involvement in manuscript preparation, loosely based on whether the statistician was listed as a coauthor, did not find any striking differences. In particular, there was no significant impact on reporting quality, concordant with one previous abstract that was limited to Indian medical journals [13]. As many items in the CONSORT guidelines are not directly related to statistics [11], we also carried out an analysis solely on the basis of the CONSORT criteria for statistical reporting and did not find any differences (data not shown). Interestingly, a trend was observed where fewer trials with a statistician listed as a coauthor were reported as positives, a finding that may warrant further study.

One limitation of our study was the low response rate of corresponding authors despite follow-up queries. This is 
Table 1 Trial characteristics depending on corresponding author response $(n=357)$

\begin{tabular}{|c|c|c|c|}
\hline Trial characteristics & Questionnaire returned $(N=107)$ & Questionnaire not returned $(N=250)$ & $P$ value \\
\hline \multicolumn{4}{|l|}{ Year published $[N(\%)]$} \\
\hline 2005 & $24(22.4 \%)$ & $67(26.8 \%)$ & 0.46 \\
\hline 2006 & $18(16.8 \%)$ & $50(20.0 \%)$ & - \\
\hline 2007 & $27(25.2 \%)$ & $44(17.6 \%)$ & - \\
\hline 2008 & $22(20.6 \%)$ & $52(20.8 \%)$ & - \\
\hline 2009 & $16(15.0 \%)$ & $37(14.8 \%)$ & - \\
\hline Industry funded $[N(\%)]$ & $54(50.5 \%)$ & $163(65.2 \%)$ & 0.013 \\
\hline \multicolumn{4}{|l|}{ Location of lead author $[N(\%)]$} \\
\hline Europe & $68(63.6 \%)$ & $146(58.4 \%)$ & 0.58 \\
\hline North America & $28(26.2 \%)$ & $79(31.6 \%)$ & - \\
\hline Other & $11(10.3 \%)$ & $25(10.0 \%)$ & - \\
\hline $3+$ Treatment arms $[N(\%)]$ & $14(13.1 \%)$ & $29(11.6 \%)$ & 0.72 \\
\hline \multicolumn{4}{|l|}{ Journal impact factor } \\
\hline$<10$ & $28(26.2 \%)$ & $67(26.8 \%)$ & 0.57 \\
\hline $10-20$ & $67(62.6 \%)$ & $145(58.0 \%)$ & - \\
\hline$>20$ & $12(11.2 \%)$ & $38(15.2 \%)$ & - \\
\hline \multicolumn{4}{|l|}{ Primary outcome results $[N(\%)]$} \\
\hline Positive & $43(40.2 \%)$ & $106(42.4 \%)$ & 0.76 \\
\hline Negative & $59(55.1 \%)$ & $136(54.4 \%)$ & - \\
\hline Unclear & $5(4.7 \%)$ & $8(3.2 \%)$ & - \\
\hline \multicolumn{4}{|l|}{ Author conclusions [N (\%)] } \\
\hline Positive & $55(51.4 \%)$ & $130(52.0 \%)$ & 0.79 \\
\hline Negative & $47(43.9 \%)$ & $112(44.8 \%)$ & - \\
\hline Unclear & $5(4.7 \%)$ & $8(3.2 \%)$ & - \\
\hline Median (range) sample size & $401(56-8028)$ & $445(42-5081)$ & 0.33 \\
\hline Median (range) 2001 CONSORT OOS & $13(12-15)$ & $14(12-15)$ & 0.33 \\
\hline Median (range) 2010 CONSORT OQS & $19(17-21)$ & $19(17-21)$ & 0.16 \\
\hline \multicolumn{4}{|l|}{ Statistician responsible for RCT design [N (\%)] } \\
\hline Included as publication coauthor & $73(68 \%)$ & - & - \\
\hline Grade $\mathrm{PhD}$ or equivalent & $78(73 \%)$ & - & - \\
\hline \multicolumn{4}{|l|}{ Statistician responsible for RCT analysis [N (\%)] } \\
\hline Included as publication coauthor & $87(81 \%)$ & - & - \\
\hline Grade $\mathrm{PhD}$ or equivalent & $72(67 \%)$ & - & - \\
\hline Trial correctly reported as positive trial ${ }^{a}[N(\%)]$ & $43(40 \%)$ & $107(43 \%)$ & 0.81 \\
\hline Trial incorrectly reported as positive by authors ${ }^{\mathrm{b}}[N(\%)]$ & $12(11 \%)$ & $24(10 \%)$ & 0.70 \\
\hline
\end{tabular}

Proportions were compared using the $\chi^{2}$-test. Continuous variables were compared using the Mann-Whitney $U$-test.

CONSORT, Consolidated Standards of Reporting Trial; OQS, Overall Reporting Quality Score; RCT, randomized clinical trial.

${ }^{a}$ On the basis of the information provided in the publication, the study reviewer concurred that the study was a positive study.

${ }^{b}$ On the basis of the information provided in the publication, the study reviewer concluded that the results were unclear or negative.

Table 2 Factors associated with statistician authorship and highest degree of education

\begin{tabular}{|c|c|c|c|c|c|c|}
\hline \multirow[b]{2}{*}{ Trial characteristics } & \multicolumn{6}{|c|}{ Statistician involved in the RCT design } \\
\hline & $\begin{array}{c}\text { Named coauthor } \\
\qquad N=73\end{array}$ & $\begin{array}{l}\text { Unnamed collaborator } \\
\qquad N=34\end{array}$ & $P$ value & $\begin{array}{c}\mathrm{PhD} \\
N=78\end{array}$ & $\begin{array}{c}\text { Other } \\
N=29\end{array}$ & $P$ value \\
\hline Trial correctly reported as positive trial ${ }^{\mathrm{a}}[N(\%)]$ & $26(36 \%)$ & $17(50 \%)$ & 0.20 & $32(41 \%)$ & $10(34 \%)$ & 0.66 \\
\hline Trial incorrectly reported as positive by authors ${ }^{b}[N(\%)]$ & $7(10 \%)$ & $5(15 \%)$ & 0.51 & $7(9 \%)$ & $4(14 \%)$ & 0.48 \\
\hline Median (IQR) 2001 CONSORT OQS & $13(12-15)$ & $13(12-15)$ & 0.78 & $13(12-15)$ & $14(13-15)$ & 0.13 \\
\hline Median (IQR) 2010 CONSORT OQS & $\begin{array}{c}19(17-21) \\
N=87\end{array}$ & $\begin{array}{c}19(18-21) \\
N=20\end{array}$ & $\begin{array}{l}0.88 \\
P \text { value }\end{array}$ & $\begin{array}{c}19(17-21) \\
N=72\end{array}$ & $\begin{array}{c}19(18-22) \\
N=35\end{array}$ & $\begin{array}{c}0.36 \\
P \text { value }\end{array}$ \\
\hline Trial correctly reported as positive trial ${ }^{a}[N(\%)]$ & $31(36 \%)$ & $12(60 \%)$ & 0.075 & $26(36 \%)$ & $16(46 \%)$ & 0.40 \\
\hline Trial incorrectly reported as positive by authors ${ }^{b}[N(\%)]$ & $10(11 \%)$ & $2(10 \%)$ & 1.00 & $7(10 \%)$ & $4(11 \%)$ & 0.75 \\
\hline Median (IQR) 2001 CONSORT OQS & $14(12-15)$ & $13(12-15)$ & 0.39 & $13(12-15)$ & $14(13-15)$ & 0.13 \\
\hline Median (IQR) 2010 CONSORT OQS & $19(18-21)$ & $18(16-20)$ & 0.22 & $19(17-21)$ & $19.5(18-22)$ & 0.23 \\
\hline
\end{tabular}

CONSORT, Consolidated Standards of Reporting Trial; IQR, interquartile range; OQS, Overall Reporting Quality Score; RCT, randomized clinical trial.

aOn the basis of the information provided in the publication, the study reviewer concurred that the study was a positive study.

${ }^{b}$ On the basis of the information provided in the publication, the study reviewer concluded that the results were unclear or negative.

a recognized problem [14] and the response rate obtained was similar to those of similar studies [15]. The response rate was lower among corresponding authors of industryfunded RCTs. This observation can be possibly explained by staff turnover or outsourcing of different aspects of RCTs. Alternatively, corresponding authors unaware of who was responsible for statistical support, or where no such person existed, may have been less likely to respond, resulting in a potential overestimate of these results. In addition, all RCTs included in this survey were moderate to large in size, multiarm, included the use of a systemic anticancer therapy, and had been previously published in a high-quality, peer-reviewed journal. It is possible that RCTs included in this review are in general of higher quality or were more likely to have a greater level of statistician involvement than smaller, single-arm, 
or unpublished studies. This could explain the high degree levels of most statisticians involved in design and analysis and the high frequency of their presence among coauthors. This imbalance could explain the lack of a significant difference observed in OQS between trials where statisticians held a $\mathrm{PhD}$ or not, and between trials where statisticians were listed as coauthors or not. In addition, as part of the journal review process, a statistician reviewer may be involved who could affect the study quality. Finally, it is recognized that coauthorship and educational degree are not perfect surrogates for statistician involvement and expertise; certainly, many non- $\mathrm{PhD}$ level statisticians are very experienced and provide superior statistical abilities, whereas many investigators may rely on statistical consultants or a group of statisticians who individually do not qualify for coauthor recognition.

Overall, we provide the first estimate of the frequency with which statisticians are involved in the reporting of trials. Although methodological limits do not allow a conclusive statement that involvement of a qualified statistician improves study quality, this study does highlight the need for greater accessibility and disclosure. A case could be made that all studies should indicate one individual as primarily responsible for the statistical study design, analysis, and reporting, especially given the recent emphasis of adequate statistical reporting by many journals, including defining coauthor roles and requiring complete protocols to be provided with submission.

\section{Acknowledgements Conflicts of interest}

There are no conflicts of interest.

\section{References}

1 Charles P, Giraudeau B, Dechartres A, Baron G, Ravaud P. Reporting of sample size calculation in randomised controlled trials: review. BMJ 2009; 338:b1732.

2 Lai R, Chu R, Fraumeni M, Thabane L. Quality of randomized controlled trials reporting in the primary treatment of brain tumors. J Clin Oncol 2006; 24:1136-1144.

3 Rios LP, Odueyungbo A, Moitri MO, Rahman MO, Thabane L. Quality of reporting of randomized controlled trials in general endocrinology literature. J Clin Endocrinol Metab 2008; 93:3810-3816.

4 Toulmonde M, Bellera C, Mathoulin-Pelissier S, Debled M, Bui B, Italiano A. Quality of randomized controlled trials reporting in the treatment of sarcomas. J Clin Oncol 2011; 29:1204-1209.

5 Peron J, Pond GR, Gan HK, Chen EX, Almufti R, Maillet D, et al. Quality of reporting of modern randomized controlled trials in medical oncology, a systematic review. J Nat/ Cancer Inst 2012; 104:982-989.

6 Carroll KJ. Back to basics: explaining sample size in outcome trials, are statisticians doing a thorough job? Pharm Stat 2009; 8:333-345.

7 Halpern SD, Karlawish JH, Berlin JA. The continuing unethical conduct of underpowered clinical trials. JAMA 2002; 288:358-362.

8 Simon R. The role of statisticians in intervention trials. Stat Methods Med Res 1999; 8:281-286.

9 International Committee of Medical Journal Editors. Uniform Requirements for Manuscripts Submitted to Biomedical Journals: Writing and Editing for Biomedical Publication. 2010. Available at: http://www.icmje.org/ urm_full.pdf [Accessed 20 November 2012].

10 Moher D, Hopewell S, Schulz KF, Montori V, Gotzsche PC, Devereaux PJ, et al. CONSORT 2010 explanation and elaboration: updated guidelines for reporting parallel group randomised trials. BMJ 2010; 340:c869.

11 Moher D, Schulz KF, Altman DG. The CONSORT statement: revised recommendations for improving the quality of reports of parallel-group randomised trials. Lancet 2001; 357:1191-1194.

12 You B, Gan H, Pond G, Chen XE. Consistency in the analysis and reporting of primary endpoints in oncology randomized controlled trials from registration to publication: a systematic review. J Clin Oncol 2012; 30: 210-216.

13 Jaykaran Chavda N, Yadav P. Proper reporting of statistical parameters in clinical trials published in Indian medical journals. Is inclusion of statistician play any significant role? J Young Pharm 2011; 3:167-168.

14 Wren JD, Grissom JE, Conway T. E-mail decay rates among corresponding authors in MEDLINE. EMBO Rep 2006; 7:122-127.

15 Sheehan K. E-mail survey response rates: a review. J Comput Mediat Commun 2001; 6:Available at: http://jcmc.indiana.edu/vo/6/issue2/ sheehan.htm/ [Accessed 26 November 2012]. 